\title{
Is Higher Roasting of Coffee Less Beneficial to Health?
}

\author{
Suminori Kono* \\ MedStat Corporation, Hakata-ku, Fukuoka 812-0023, Japan \\ *Corresponding author: Suminori Kono, MedStat Corporation, Hakata-ku, Fukuoka 812-0023, Japan
}

\begin{abstract}
ARTICLE INFO
Received: 幽 August 05, 2019

Published: August 09, 2019

Citation: Suminori Kono. Is Higher Roasting of Coffee Less Beneficial to

ABSTRACT

Coffee drinking has been related to decreased risks of chronic diseases. Chlorogenic acids (CGAs) are not only potent antioxidants in coffee brew, but also have other potentially beneficial effects. Loss of CGAs due to higher roasting raises a concern with respect to health benefit conferred by coffee drinking. In this review, the author summarized recent findings regarding the changes in coffee compounds by roasting and discussed implication of the decrease in CGAs associated with roasting in relation to human health
\end{abstract} Health?. Biomed J Sci \& Tech Res 20(3)2019. BJSTR. MS.ID.003452.

Keywords: Coffee; Chlorogenic Acids; Health Effects; Roasting
Abbreviations: CGAs: Chlorogenic Acids, 5-CQA: 5-O-Caffeoylquinic Acid, ORAC: Oxygen Radical Absorbance Capacity

\section{Introduction}

Coffee drinking has been related to decreased risks of chronic diseases. Most consistent findings are decreased risks of type 2 diabetes mellitus, liver diseases of various stages, and Parkinson's disease associated with coffee drinking [1]. Coffee brew contains a wide variety of bioactive compounds. Caffeine and chlorogenic acids (CGAs) are coffee compounds that have been studied most intensively with respect to occurrence, metabolism, and physiological effects [2,3]. Roasting coffee beans confers different flavors, tastes, and colors to coffee brew according to the extent of roasting. Roasting also alters the composition of coffee compounds, decreasing the content of CGAs, while it does not change caffeine content [2,3]. CGAs are not only potent antioxidants in coffee brew, but also have other potentially beneficial effects against carcinogenesis and neuronal degeneration, anti-platelet effect in relation to cardiovascular protection, and antidiabetic effects on glucose metabolism [1,2]. Thus, loss of CGAs due to higher roasting raises a concern with respect to health benefit conferred by coffee drinking. In this review, the author summarized recent findings regarding the changes in coffee compounds by roasting and discussed implication of the decrease in CGAs associated with roasting in relation to human health.

\section{Coffee Roasting and Composition Changes}

CGAs in coffee are primarily hydroxycinnamic acids (typically, caffeic acid and ferulic acid) esterified with quinic acid. The most abundant CGA in coffee is 5-0-caffeoylquinic acid (5-CQA) followed by its isomers 3-CQA and 4-CQA. Dicaffeoylquinic acid (3,4-diCQA, 3,5-diCQA, and 4,5-diCQA) and feruloylquinic acids (3-FQA, 4-FQA, and 5-FQA) are also among coffee CGAs [3]. According to the conventional American classification [4], roasting level is classified into light (lowest level), cinnamon, medium, high, city, full city, French, and Italian (highest level). These are often collapsed into broader categories: light roast (light and cinnamon), medium roast (medium and high), medium-dark roast (city and full city), and dark roast (French and Italian). The content of CGAs in coffee brew decreases progressively with increasing levels of roast. In an experiment [5], light, medium, city, and French roasting reduced the content of CGAs in green coffee to $50 \%, 30 \%, 5 \%$, and $<1 \%$, respectively. In another experiment [6], the content of CGAs in green coffee decreased to $109 \%, 77 \%, 42 \%, 18 \%, 10 \%$, and $5 \%$, respectively, after roasting of 5 (very light), 6, 7, 8, 9, and 10-15 minutes (very dark) at the maximum temperature of $230^{\circ} \mathrm{C}$.

The percent changes were calculated by averaging the reported amounts for multiple species. It may be strange that the total amount of CGAs increased by $9 \%$ at 5 minutes of roasting in the latter study [6]. This is largely because of increases of 3-CQA and 4-CQA which are resulted from isomerization (acyl migration) and hydrolysis of other CGAs [3]. In an experiment based on a more natural extraction [7], coffee brew prepared by dark roast coffee contained one third of the amount of CQA (sum of 3-CQA, 4-CQA, and 5-CQA) in coffee brew of light roast coffee. Chemical reactions during roasting also produce CGA lactones and quinic lactone 
(quinide). The amount of CGA lactones was progressively higher up to roasting of less than 8 minutes, but the content was lower in the extract from coffee beans roasted longer [6].

Another reaction linked to roasting is the Maillard reaction, by which melanoidins are produced via reaction of reducing sugars with amino acids or proteins. Incorporation of CGAs into melanoidins is another reason for the loss of CGAs due to roasting. Melanoidins have antioxidant and metal chelating activity and other bioactive functions, and thus melanoidins may compensate the decrease in CGAs resulted from roasting. Antioxidant activities of whole coffee brew decreased progressively with increasing levels of roasting, however [8]. High-molecular weight fraction of roasted coffee brew, which probably represented phenols incorporated into melanoidins, showed higher antioxidant activities than that of green coffee, but the activity did not differ by degree of roasting [8]. It was also estimated that melanoidin-bound phenols accounted for $25-47 \%$ of the antioxidant activity. Roasting process degrades trigonelline partially to nicotinic acid and several pyridine derivatives; trigonelline has been implicated as exerting a protective effect on glucose metabolism [2]. Roasting does not seem to materially affect the content of diterpenes (kahweol and cafestol) [9], which seem to have potent hepatoprotective properties [10] although they are known to elevate blood cholesterol levels [2].

\section{Roasting and Biological Effects}

Few studies have addressed differential biological effects of coffee according to roasting levels. Effects against lipopolysaccharide-induced inflammation in mice did not differ in pretreatment with coffee brew of light, medium, city, and French roast [11]. Roasted coffee rather than green coffee was shown to be more neuroprotective against oxidative stress in an in vitro study of neuronal cell culture [12]. Interesting, the study also showed that lipophilic oxygen radical absorbance capacity (ORAC) was markedly higher in roasted coffee extract than in green coffee extract, while there was no difference in hydrophilic ORAC between roasted and green coffee. There are no human studies showing that light roast coffee is better than dark roast coffee. Of particular interest are epidemiological studies in Italy where dark roast coffee is commonly consumed as espresso coffee. A substantial number of Italian studies have reported protective associations between coffee and liver diseases [10], while no study has addressed the association between coffee and type 2 diabetes in Italy. Espresso coffee was related to an increased risk of coronary heart disease in a prospective study [13]. Espresso coffee contains a larger amount of diterpenes than filtered coffee, but espresso coffee was unrelated to blood cholesterol [13]. The finding is rather difficult to interpret, but it is unlikely that dark roast coffee is harmful with respect to coronary heart disease.

\section{Conclusion}

Higher degree of roasting results in a substantial decrease in the content of CGAs which represent bioactive compounds in coffee brew. The overall antioxidant capacity of coffee brew also decreases with increasing levels of roasting. Nonetheless, roasted coffee may be more neuroprotective than green coffee. There is no epidemiological evidence that heavily roasted coffee such as espresso coffee is less beneficial to human health. It is notable that one serving of espresso coffee purchased in Italy contains almost as large an amount of CQA as a cup of instant coffee [7].

\section{References}

1. Poole R, Kennedy OJ, Roderick P, Fallowfield JA, Hayes PC, et al. (2017) Coffee consumption and health: umbrella review of meta-analyses of multiple health outcomes. BMJ 359: j5024.

2. Ludwig IA, Clifford MN, Lean ME, Ashihara H, Crozier A, et al. (2014) Coffee: biochemistry and potential impact on health. Food Funct 5(8): 1695-1717.

3. Clifford MN, Jaganath IB, Ludwig IA, Crozier A (2017) Chlorogenic acids and the acyl-quinic acids: discovery, biosynthesis, bioavailability and bioactivity. Nat Prod Rep 34(12): 1391-1421.

4. (2019) National Coffee Association of U.S.A Inc. Coffee Roast Guide.

5. Moon J K, Yoo HS, Shibamoto T (2009) Role of roasting conditions in the level of chlorogenic acid content in coffee beans: Correlation with coffee acidity. J Agric Food Chem 57(12): 5365-5369.

6. Farah A, de Paulis T, Trugo LC, Martin PR (2005) Effect of roasting on the formation of chlorogenic acid lactones in coffee. J Agric Food Chem 53(5): 1505-1513.

7. Ludwig IA, Mena P, Calani L, Cid C, Del Rio D, et al. (2014) Variations in caffeine and chlorogenic acid contents of coffees: what are we drinking? Food Funct 5(8): 1718-1726.

8. Perrone D, Farah A, Donangelo CM (2012) Influence of coffee roasting on the incorporation of phenolic compounds into melanoidins and their relationship with antioxidant activity of the brew. J Agric Food Chem 60(17): 4265-4275.

9. Williamson K, Hatzakis E (2019) Evaluating the effect of roasting on coffee lipids using a hybrid targeted-untargeted NMR approach in combination with MRI. Food Chem 299: 125039.

10. Heath RD, Brahmbhatt M, Tahan AC, Ibdah JA, Tahan V, et al. (2017) Coffee: The magical bean for liver diseases. World J Hepatol 9(15): 689696.

11. Choi S, Jung S, Ko KS (2018) Effects of coffee extracts with different roasting degrees on antioxidant and anti-inflammatory systems in mice. Nutrients $10(3)$ pii: E363.

12. Chu YF, Brown PH, Lyle BJ, Chen Y, Black RM, et al. (2009) Roasted coffees high in lipophilic antioxidants and chlorogenic acid lactones are more neuroprotective than green coffees. J Agric Food Chem 57(20):98019808.

13. Grioni S, Agnoli C, Sieri S, Pala V, Ricceri F, et al. (2015) Espresso coffee consumption and risk of coronary heart disease in a large Italian cohort. PLoS One 10(5): e0126550. 
ISSN: 2574-1241

DOI: 10.26717/BJSTR.2019.20.003452

Suminori Kono. Biomed J Sci \& Tech Res

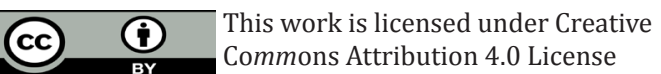

Submission Link: https://biomedres.us/submit-manuscript.php

$\begin{array}{ll}\text { BIOMEDICAL } & \text { Assets of Publishing with us } \\ \text { RESEARCHES } & \text { - Global archiving of articles } \\ & \text { - Immediate, unrestricted online access } \\ \end{array}$

